Dongen, B. [et al.]. Aligning modeled and observed behavior: A compromise between computation complexity and quality. A: International Conference on Advanced Information Systems Engineering. "Advanced Information Systems Engineering, 29th International Conference, CAiSE 2017: Essen, Germany, June 12-16, 2017: proceedings". Berlín: Springer, 2017, p. 94-109. The final authenticated version is available online at https://doi.org/10.1007/978-3-319-59536-8_7

\title{
Aligning Modeled and Observed Behavior: A Compromise Between Complexity and Quality
}

\author{
Boudewijn van Dongen ${ }^{1}$, Josep Carmona ${ }^{2}$, Thomas Chatain ${ }^{3}$, and Farbod Taymouri ${ }^{2}$ \\ 1 Eindhoven University of Technology, The Netherlands \\ b.f.v.dongen@tue.nl \\ 2 Universitat Politècnica de Catalunya, Barcelona (Spain) \\ \{jcarmona, taymouri\}@cs.upc.edu \\ 3 LSV, ENS Cachan, CNRS, INRIA, Université Paris-Saclay, Cachan (France) \\ chatainelsv.ens-cachan.fr
}

\begin{abstract}
Certifying that a process model is aligned with the real process executions is perhaps the most desired feature a process model may have: aligned process models are crucial for organizations, since strategic decisions can be made easier on models instead of on plain data. In spite of its importance, the current algorithmic support for computing alignments is limited: either techniques that explicitly explore the model behavior (which may be worst-case exponential with respect to the model size), or heuristic approaches that cannot guarantee a solution, are the only alternatives. In this paper we propose a solution that sits right in the middle in the complexity spectrum of alignment techniques; it can always guarantee a solution, whose quality depends on the exploration depth used and local decisions taken at each step. We use linear algebraic techniques in combination with an iterative search which focuses on progressing towards a solution. The experiments show a clear reduction in the time required for reaching a solution, without sacrificing significantly the quality of the alignment obtained.
\end{abstract}

\section{Introduction}

The current trend to store all kinds of digital data has made organizations to become more than ever data-oriented, thus dependent on the available techniques to extract value from the data. Process mining is an emerging field which focuses on analyzing the data corresponding to process executions, with the purpose of extracting, analyzing and enhancing evidence-based process models [1]. The application of process mining techniques is magnified in the field of Business Process Management, where in the last couple of years we have seen important vendors incorporating process mining capabilities to their products ${ }^{4}$.

One of the current challenges for process mining techniques is the computation of an alignment of a process model with respect to observed behavior [2]. Intuitively, given a trace representing a real process execution, an optimal alignment provides the best trace the process model can provide to mimic the observed behavior. Then observed and

\footnotetext{
${ }^{4}$ http://go.sap.com/product/technology-platform/process-mining. html 
model traces are rendered in a two-row matrix denoting the synchronous/asynchronous moves between individual activities of model and log, respectively. Alignments are extremely important in the context of process mining, since they open the door to evaluate the metrics that asses the quality of a process model to represent observed behavior: fitness and generalization [2] and precision [3]. Additionally, alignments are a necessary step to enhance the information provided in a process model [1].

The current algorithmic support to compute alignments is either too complex [2] or heuristic [4]. The former is defined as a search for a minimal path on the product of the state space of the process model and the observed behavior, an object that is worstcase exponential with respect to the size of the model. This hampers the application of the techniques from [2] in case of medium/large instances. In contrast, the techniques in [4] are very efficient both in time and memory requirements, but cannot guarantee a solution always.

This paper presents an algorithm for computing alignments whose nature is in between the two aforementioned techniques. As in [4], we ground the technique on the resolution of Integer Linear Programming (ILP) models that guide the search for solutions while constructing the derived alignment. However, the techniques of this paper ensure the derivation of an alignment by requiring the feasibility of individual steps computed, in contrast to the recursive approach applied in [4]. As in [2], the algorithm is defined on the synchronous product between the observed trace and the process model, and we use part of the ILP model (the tail of the solutions obtained at each step) as an underestimate of the cost to reach a solution. The approach is implemented in the open-source platform ProM, and experiments are provided which witness the distinctive capabilities of the proposed approach with respect to the state-of-the-art technique to compute alignments.

\subsection{Related Work}

The seminal work in [2] proposed the notion of alignment, and developed a technique to compute optimal alignments for a particular class of process models. For each trace $\sigma$ in the $\log$, the approach consists on exploring the synchronous product of model's state space and $\sigma$. In the exploration, the shortest path is computed using the $A^{*}$ algorithm, once costs for model and log moves are defined. The approach is implemented in ProM, and can be considered as the state-of-the-art technique for computing alignments. Several optimizations have been proposed to the basic approach: for instance, the use of ILP techniques on each visited state to prune the search space [2]. In contrast to [2], the technique presented in [4] fully resorts in the resolution of ILP models together with a recursive partitioning of the input trace. This technique computes approximate alignments, a novel class of alignments where deviations can be explained between sets of transitions, instead of singletons as in [2]. The techniques in [4] can be a good alternative when a precise information is not required and instead an approximation suffices.

Decompositional techniques have been presented $[5,6]$ that instead of computing alignments, they focus on the decisional problem of whereas a given trace fits or not a process model. The underlying idea is to split the model into a particular set of transition-bordered fragments which satisfy certain conditions, and local alignments can be computed for each one of the fragments, thus providing a upper bound on the 
cost of an alignment. In contrast, the technique presented in this paper does not split the model, hence enabling the computation of alignments at a global (model) level.

\section{Preliminaries}

\subsection{Petri nets}

A Petri Net [7] is a 3-tuple $N=\langle P, T, \mathcal{F}\rangle$, where $P$ is the set of places, $T$ is the set of transitions, $P \cap T=\emptyset, \mathcal{F}:(P \times T) \cup(T \times P) \rightarrow\{0,1\}$ is the flow relation. A marking is an assignment of non-negative integers to places. If $k$ is assigned to place $p$ by marking $m$ (denoted $m(p)=k$ ), we say that $p$ is marked with $k$ tokens. Given a node $x \in P \cup T$, its pre-set and post-set (in graph adjacency terms) are denoted by ${ }^{\bullet} x$ and $x^{\bullet}$ respectively. A transition $t$ is enabled in a marking $m$ when all places in ${ }^{\bullet} t$ are marked. When a transition $t$ is enabled, it can fire by removing a token from each place in $\bullet$ and putting a token to each place in $t^{\bullet}$. A marking $m^{\prime}$ is reachable from $m$ if there is a sequence of firings $t_{1} t_{2} \ldots t_{n}$ that transforms $m$ into $m^{\prime}$, denoted by $m\left[t_{1} t_{2} \ldots t_{n}\right\rangle m^{\prime}$. A sequence of transitions $t_{1} t_{2} \ldots t_{n}$ is a feasible sequence if it is firable from the initial marking $m_{0}$.

Workflow processes can be represented in a simple way by using Workflow Nets (WF-nets). A WF-net is a Petri net where there is a place start (denoting the initial state of the system) with no incoming arcs and a place end (denoting the final state of the system) with no outgoing arcs, and every other node is within a path between start and end. The transitions in a WF-net are labeled with tasks or are used for routing purposes (so-called silent transitions or $\tau$ transitions). For the sake of simplicity, the techniques of this paper assume models are specified with sound labeled WF-nets, i.e. models without lifelocks and with only a single deadlock indicating that the model's execution has terminated.

Definition 1 (Net System, Full Firing Sequences). A net system is a tuple $S N=$ $\left(N, m_{\text {start }}, m_{\text {end }}\right)$, where $N$ is a Petri net and the two last elements define the initial and final marking of the net, respectively. The set $\left\{\sigma \mid\left(N, m_{\text {start }}\right)[\sigma\rangle\left(N, m_{\text {end }}\right)\right\}$ denotes all the full firing sequences of $S N$.

Note that in this paper, we assume that the set of all full firing sequences is not empty, i.e. the final marking is reachable from the initial marking.

\subsection{Petri nets and Linear Algebra}

Let $N=\langle P, T, \mathcal{F}\rangle$ be a Petri net with initial marking $m_{0}$. Given a feasible sequence $m_{0} \stackrel{\sigma}{\rightarrow} m$, the number of tokens for a place $p$ in $m$ is equal to the tokens of $p$ in $m_{0}$ plus the tokens added by the input transitions of $p$ in $\sigma$ minus the tokens removed by the output transitions of $p$ in $\sigma$ :

$$
m(p)=m_{0}(p)+\sum_{t \in \bullet}|\sigma|_{t} \mathcal{F}(t, p)-\sum_{t \in p^{\bullet}}|\sigma|_{t} \mathcal{F}(p, t)
$$

The marking equations for all the places in the net can be written in the following matrix form: $m=m_{0}-\mathbf{N}^{-} \cdot \widehat{\sigma}+\mathbf{N}^{+} \cdot \widehat{\sigma}$, where $\mathbf{N}=\mathbf{N}^{+}-\mathbf{N}^{-} \in \mathbb{Z}^{P \times T}$ is the 
incidence matrix of the net: $\mathbf{N}^{-}(p, t)=\mathcal{F}(p, t)$ corresponds to the consumption of tokens and $\mathbf{N}^{+}(p, t)=\mathcal{F}(t, p)$ corresponds to production of tokens. If a marking $m$ is reachable from $m_{0}$, then there exists a sequence $\sigma$ such that $m_{0} \stackrel{\sigma}{\rightarrow} m$, and the following system of equations has at least the solution $\vec{x}=\widehat{\sigma}$

$$
\vec{m}=\overrightarrow{m_{0}}-\mathbf{N}^{-} \cdot \vec{x}+\mathbf{N}^{+} \cdot \vec{x}
$$

If (1) is infeasible, then $m$ is not reachable from $m_{0}$. The inverse does not hold in general: there are markings satisfying (1) which are not reachable. Those markings (and the corresponding Parikh vectors) are said to be spurious [8].

For well-structured Petri nets classes equation (1) characterizes reachability. It goes beyond the scope of this paper to elaborate on the exact classes of models for which this is the case. However, in this paper, we assume that the models we consider belong to this class.

\subsection{Foundations of Alignments}

Definition 2 (Trace, Event Log, Parikh vector). Given an alphabet of events $T=$ $\left\{t_{1}, \ldots, t_{n}\right\}$, a trace is a word $\sigma \in T^{*}$ that represents a finite sequence of events. An event $\log L \in \mathcal{B}\left(T^{*}\right)$ is a multiset of traces ${ }^{5} .|\sigma|_{a}$ represents the number of occurrences of a in $\sigma$. The Parikh vector of a sequence of events $\sigma$ is a function $\widehat{\wedge}^{*} T^{*} \rightarrow \mathbb{N}^{n}$ defined as $\widehat{\sigma}=\left(|\sigma|_{t_{1}}, \ldots,|\sigma|_{t_{n}}\right)$. For simplicity, we will also represent $|\sigma|_{t_{i}}$ as $\widehat{\sigma}\left(t_{i}\right)$.

The main metric in this paper to asses the adequacy of a model in describing a log is fitness [1], which is based on the reproducibility of a trace in a model:

Definition 3 (Fitting Trace). A trace $\sigma \in T^{*}$ fits $S N=\left(N, m_{\text {start }}, m_{\text {end }}\right)$ if $\sigma$ coincides with a full firing sequence of $S N$, i.e., $\left(N, m_{\text {start }}\right)[\sigma\rangle\left(N, m_{\text {end }}\right)$.

Hence an optimal alignment may be fitting or not, depending on whether the model can mimic exactly or not the behavior observed. Computing alignments is a complex task. In [2] the foundational work was presented to construct alignments by depth-first search using an $A^{*}$ algorithm. The algorithm presented there relies on two fundamental concepts:

- A synchronous product Petri net, which is a combination of the original model being aligned and a Petri net representation of the (partially ordered) trace in the $\log$, and

- The marking equation of that synchronous product.

The core alignment question is formalized as follows: Given a synchronous product with a penalty function assigning a non-negative penalty to each transition firing, find a firing sequence from the initial marking to the final marking with the lowest total penalties.

Consider the example model in Figure 1. This model is a simple parallelism between transitions $B$ and $C$ after $A$ and before $D$. Now, consider the trace $\langle C, D\rangle$

\footnotetext{
${ }^{5} \mathcal{B}(A)$ denotes the set of all multisets of the set $A$.
} 


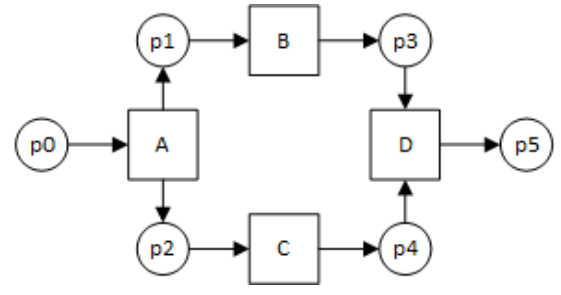

Fig. 1. Example model.

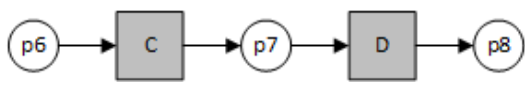

Fig. 2. Example trace net.

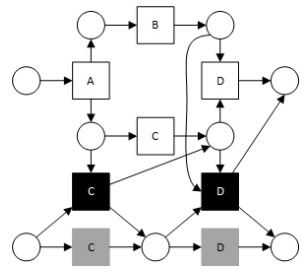

Fig. 3. Example Synchronous Product.

\begin{tabular}{c|c|c|c}
$\mathrm{A}$ & $\mathrm{C}$ & $\mathrm{B}$ & $\mathrm{D}$ \\
\hline $\mathrm{P}$ & $\mathrm{C}$ & $\mathrm{D}$ & $\mathrm{D}$
\end{tabular}

Fig. 4. An optimal alignment.

translated into a trace net as shown in Figure 2. Obviously, this trace does not fit the model, as transitions $A$ and $B$ are missing from it. Conceptually, the alignment problem first constructs a so-called synchronous product which is shown in Figure 3. Here, the two black transitions are synchronous combinations of equally labeled transitions in the model and the trace, i.e. they have the same input and output places in both the model and the trace net. The alignment algorithm then finds the shortest execution sequence from the initial state to the final state, where the firing of each transition has an associated cost. Typically, the black transitions, called synchronous moves have the lowest cost, while the model transitions, called model moves and the trace net transitions, called log moves, have higher costs. For this example, the cheapest firing sequence would be $\langle A, C, B, D\rangle$ as depicted in the upper row (model trace) of the alignment of Figure 4. For this alignment, the white transitions $A$ and $B$ have been fired as model moves, and the black transitions $C$ and $D$ have fired as synchronous moves.

The marking equation used for the example synchronous product model in Figure 3 is shown below. Here, the columns corresponding to each transition in the incidence matrix are labeled with $m, s$, or $l$ for (m)odel, (s)ynchronous, or (log) move.

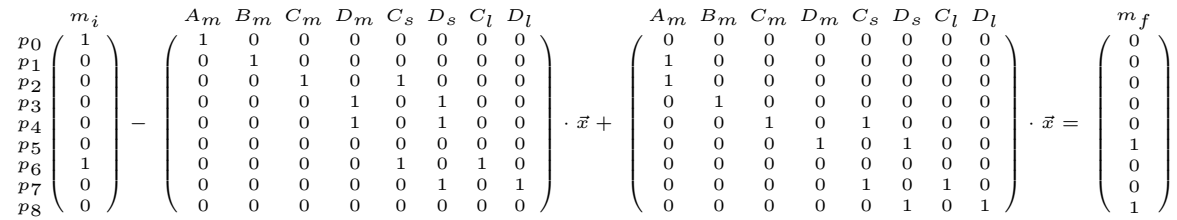

In the remainder of the paper, we consider the synchronous product model as the starting point and we use the partitioning of the transitions into synchronous moves, log moves and model moves. 
Definition 4 (Alignments, Optimal Alignments). Let $N=\langle P, T, \mathcal{F}\rangle$ be a synchronous product Petri net where $T=T^{s} \cup T^{l} \cup T^{m}$ can be partitioned into sets of transitions corresponding to synchronous moves, log moves and model moves respectively and let $\left(N, m_{\perp}, m^{\top}\right)$ a corresponding net system. Furthermore let $c: T \rightarrow \mathbb{R}^{+} a$ cost function. An alignment is a full firing sequence $\sigma_{a} \in\left\{\sigma \mid\left(N, m_{\perp}\right)[\sigma\rangle\left(N, m^{\top}\right)\right\}$ of this system. An optimal alignment is an alignment $\sigma_{a}$ such that for all $\sigma \in\{\sigma \mid$ $\left.\left(N, m_{\perp}\right)[\sigma\rangle\left(N, m^{\top}\right)\right\}$ holds that $c\left(\sigma_{a}\right) \leq c(\sigma)$.

Traditional algorithms search for alignments using a depth-first search method over a search graph in which each node represents a partial firing sequence of the system and each edge the firing of a transition.

Definition 5 (Search space). Let $N=\langle P, T, \mathcal{F}\rangle$ be a synchronous product Petri net where $T=T^{s} \cup T^{l} \cup T^{m}$ can be partitioned into sets of transitions corresponding to synchronous moves, log moves and model moves respectively and let $\left(N, m_{\perp}, m^{\top}\right)$ a corresponding net system. Furthermore let $c: T \rightarrow \mathbb{R}^{+}$a cost function. The alignment search space is defined as $S=(V, E, c)$, with $V=\left\{m \mid\left(N, m_{\perp}\right)[\sigma\rangle(N, m)\right\}$ and $E \subseteq V \times T \times V$ such that $\left(m, t, m^{\prime}\right) \in E$ if and only if $(N, m)[t\rangle\left(N, m^{\prime}\right)$. The root of the search space is $m_{\perp} \in V$ the initial marking. The target node in the search space is the final marking $m^{\top} \in V$. Note that $m^{\top} \in V$ since the final marking of a system net is assumed to be reachable.

Note that, in the general case, the search space is not bounded. There may be infinitely many markings reachable from the initial marking and hence in the search space. Finding an optimal alignment is translated as finding a shortest path from $m_{\perp}$ to $m^{\top}$ in the search space, where $c$ represents the length of the edges ${ }^{6}$.

In order to find the shortest path ${ }^{7}$ in the search space, traditional alignment approaches use the $A^{*}$ algorithm. This algorithm relies on a estimate function that underestimates the remaining costs from the current node to one of the target nodes. The cost between nodes $m$ and $m^{\prime}$ in $V$ can be underestimated by the marking equation (cf. Section 2.2) in the following way:

Definition 6 (Underestimating the costs). Let $S=(V, E, c)$ be a search space and $m_{c} \in V$ the current marking reached in the graph. We know that if there exists a $\sigma^{\prime}$ such that $\left(N, m_{c}\right)\left[\sigma^{\prime}\right\rangle\left(N, m^{\top}\right)$ then $m_{c}+\mathbf{N} \cdot \widehat{\sigma}^{\prime}=m^{\top}$.

Therefore, the solution to the linear problem minimize $c(\varsigma)$ such that $\overrightarrow{m_{c}}+\mathbf{N} \cdot \widehat{\varsigma}=$ $\overrightarrow{m^{\top}}$ provides an underestimate for the cost of $\sigma^{\prime}$, i.e. $c(\varsigma) \leq c\left(\sigma^{\prime}\right)$.

If no solution exists, the final marking cannot be reached, which implies that part of the search space is not relevant or in other words a correct underestimate for the remaining distance is infinite.

\footnotetext{
${ }^{6}$ Since the cost function $c$ does not allow for 0-length, there are no loops of length 0 in the graph. In the available implementations of the alignment problem, this is hidden from the end-user when instantiating the cost function, but an $\epsilon>0$ is used in the core computation.

${ }^{7}$ Note that there may be more than one shortest path. Where we talk about the shortest path, we mean any shortest path.
} 
This approach to finding alignments has been implemented in ProM and has been extensively used in many applications. However, there are two problems with this approach. Firstly, the search space can be very large (although only a finite part needs to be considered). Typically, the search space size is exponential in the size of the synchronous product model which is the product of the original model and the trace to be aligned. Secondly, computing estimates is computationally expensive. This can be done both using Linear Programming and Integer Linear Programming, where the latter provides more accurate estimates. In practice however, both techniques are equally fast as the increase in precision when doing Integer computations allows the $A^{*}$ algorithm to visit fewer nodes.

\section{ILP Techinques to Compute Alignments}

\subsection{Computing Optimal Alignments using ILP}

In this paper, we take a fundamentally different approach as we incrementally construct (possibly suboptimal) alignments. We do so, by "jumping" trough the synchronous product model in a depth-first manner until we reach the final marking. Once the final marking is reached, we terminate the search. Effectively, from a given marking, we fire a total of $x$ transitions such that these $x$ firings are locally optimal with respect to the cost function $c$ and we reach the next node in the search space, from where we continue our search. However, before discussing our algorithm, we first consider a method for computing optimal alignments of a given maximal length using the marking equation.

The marking equation allows us to formalize $x$ transition executions at once by taking the consumption matrix for each step and the marking equation for all preceding steps in the following way:

Property 1 (Marking equation for executing $x$ transitions). Let $N=\langle P, T, \mathcal{F}\rangle$ be a Petri net, $m_{0}, m_{f}$ two reachable markings of the net and let $\sigma=\left\langle t_{0}, \ldots, t_{x-1}\right\rangle$ be a trace such that $\left(N, m_{0}\right)[\sigma\rangle\left(N, m_{f}\right)$. Furthermore, for $0<i \leq x$, let $m_{i}$ be such that $\left(N, m_{0}\right)\left[\left\langle t_{0}, \ldots, t_{i}\right\rangle\right\rangle\left(N, m_{i}\right)$. Using the marking equation and general properties of transition firing, we know the following properties hold:

$-\overrightarrow{m_{f}}=\overrightarrow{m_{0}}-\mathbf{N}^{-} \cdot \widehat{\sigma}+\mathbf{N}^{+} \cdot \widehat{\sigma}$ as the sequence $\sigma$ is executable,

- for $0<i \leq x$ holds that $\overrightarrow{m_{i}}=\overrightarrow{m_{i-1}}-\mathbf{N}^{-} \cdot \widehat{\left\langle t_{i-1}\right\rangle}+\mathbf{N}^{+} \cdot \widehat{\left\langle t_{i-1}\right\rangle}$, i.e. the marking equation holds for each individual transition in the sequence,

- for $0 \leq i<x$ holds that $\overrightarrow{m_{i}}-\mathbf{N}^{-} \cdot \widehat{\sigma_{0 . .}}+\mathbf{N}^{+} \cdot \widehat{\sigma_{0 . . i-1}} \geq 0$, i.e. before firing of each transition there are sufficient tokens to fire that transition.

The properties above are fundamental properties of Petri nets and the marking equation. They give rise to a new algorithm to find alignments of a given length.

Definition 7 (Up To Length $x$ Alignment as ILP problem). Let $N=\langle P, T, \mathcal{F}\rangle$ be a synchronous product Petri net and let $\left(N, m_{\perp}, m^{\top}\right)$ a corresponding net system. Furthermore let $c: T \rightarrow \mathbb{R}^{+}$a cost function. Let $\overrightarrow{\theta_{0}}, \ldots, \overrightarrow{\theta_{x-1}}$ be a set of $x$ vectors of dimension $|T|$ as the optimal solution to the following $\{0,1\}$ ILP problem: 


$$
\begin{aligned}
& \underset{\Sigma}{\operatorname{minimize}} \quad \sum_{0 \leq i<x} c\left(\overrightarrow{\theta_{i}}\right) \\
& \text { subject to } \quad \overrightarrow{m_{\perp}}+\sum_{0 \leq j<x} \mathbf{N} \cdot \overrightarrow{\theta_{j}}=\overrightarrow{m^{\top}} \\
& \forall_{0 \leq i<x} \quad \vec{\theta}_{i} \cdot \overrightarrow{1}^{T} \leq 1 \\
& \overrightarrow{m_{\perp}}+\sum_{0 \leq j<i} \mathbf{N} \cdot \overrightarrow{\theta_{j}}-\mathbf{N}^{-} \cdot \overrightarrow{\theta_{i}} \geq 0 \\
& \forall 0<i<x \quad \overrightarrow{\theta_{i-1}} \cdot \overrightarrow{1}^{T} \geq \vec{\theta}_{i} \cdot \overrightarrow{1}^{T}
\end{aligned}
$$

An optimal solution to the problem above constitutes a full firing sequence $\sigma$ of length $l=\sum_{0 \leq i<x} \overrightarrow{\theta_{i}} \cdot \overrightarrow{1}^{T}$ of the net $N$ in the following way: for each $0 \leq i<l$ holds that $\sigma_{i}=t \equiv \vec{\theta}(t)=1$, i.e. the sequence $\sigma$ is made up of those transitions which correspond to the variables taking value 1 in this system. Note that for $l \leq i<x$ holds that $\vec{\theta}_{i} \cdot \overrightarrow{1}^{T}=0$.

The target function shown as equation 2 above sums the costs of firing transitions in the net. Equation 4 ensures that each vector corresponds to at most one firing of a transition and Equation 5 ensures that firing all transitions $t_{j}$ preceding transition $t_{i}$ from the initial marking produces sufficient tokens in every place to enable transition $t_{i}$. Equation 6 ensures that in any solution the vectors $\vec{\theta}=\overrightarrow{0}$ are grouped together and finally, Equation 3 ensures that the final marking is reached after firing at most $k$ transitions.

Before showing how the ILP definition above can be extended to find alignments up to length $k$,we first show that any optimal alignment $\sigma$ indeed corresponds to an optimal solution to this ILP for $k=|\sigma|$.

Theorem 1. Let $N=\langle P, T, \mathcal{F}\rangle$ be a synchronous product Petri net and let $\left(N, m_{\perp}, m^{\top}\right)$ a corresponding net system. Furthermore let $c: T \rightarrow \mathbb{R}^{+}$a cost function and $\sigma$ an optimal alignment of $N$. We show that there is an optimal solution to the $k$-alignment ILP for $k \geq|\sigma|$ corresponding to $\sigma$, i.e. the ILP-alignment problem provided us with optimal alignments.

Proof. The proof consists of two parts. First, we show that $\sigma$ translates into a solution of the ILP. Then, we show that there cannot be a more optimal solution as this would imply there is a more optimal alignment.

Let $\Theta=\left\{\overrightarrow{\theta_{0}}, \ldots, \overrightarrow{\theta_{|\sigma|-1}}\right\}$ be a set of vectors, such that for all $0 \leq i<|\sigma|$ holds that $\vec{\theta}_{i}(t)=1$ if and only if $\sigma_{i}=t$, otherwise $\vec{\theta}_{i}(t)=0$. We show that this is a solution to the ILP of Definition 7 by enumerating the constraints:

(4) For all $0 \leq i<\left|\sigma_{a}\right|$ it trivially holds that $\vec{\theta}_{i} \cdot \overrightarrow{1}^{T}=1$,

(5) Since $\sigma$ is a full firing sequence, we know that for each $0 \leq i<|\sigma|$ holds that $\left(N, m_{\perp}\right)\left[\sigma_{0 . . i-1}\right\rangle(N, m)$ for some marking $m$ in which transition $\sigma_{i}$ is enabled. Furthermore, the marking equation states that $\overrightarrow{m_{\perp}}+\mathbf{N} \cdot \widehat{\sigma_{0 . i-1}}=\vec{m}$ and $\vec{m}-\mathbf{N}^{-}$. $\widehat{\left\langle\sigma_{i}\right\rangle} \geq 0$. 
The definition $\overrightarrow{\theta_{i}}$ leads to the fact that $\sum_{0<j<i} \overrightarrow{\theta_{j}}=\widehat{\sigma_{0 . . i-1}}$, hence we conclude that $\overrightarrow{m_{\perp}}+\mathbf{N} \cdot \sum_{0 \leq j<i} \overrightarrow{\theta_{j}}=\vec{m}$ and $\vec{m}-\mathbf{N}^{-} \cdot \theta_{i} \geq 0$. Combining this yields $\overrightarrow{m_{\perp}}+\sum_{0 \leq j<i} \mathbf{N} \cdot \overrightarrow{\theta_{j}}-\mathbf{N}^{-} \cdot \theta_{i} \geq 0$ for all $0 \leq i<|\sigma|$,

(6) Since all vectors $\overrightarrow{\theta_{i}}$ contain one element equal to 1 this is trivially true,

(3) Similar to the proof for Equation 5, this equation is satisfied.

The set of vectors $\Theta$ indeed is a solution to the ILP corresponding to the full firing sequence $\sigma$. Now we prove that no better solution to the ILP exists by contradiction. Assume there is a solution $\Theta^{\prime}=\left\{\overrightarrow{\theta_{0}^{\prime}}, \ldots, \overrightarrow{\theta_{|\sigma|-1}^{\prime}}\right\}$ which is a solution to the ILP with a lower target function than $\Theta$. We know we can construct a $\sigma^{\prime}=\left\langle t_{0}, \ldots, t_{l-1}\right\rangle$ for $\Theta^{\prime}$ with length $l \leq|\sigma|$ (Definition 7). Furthermore, we know $\sigma^{\prime}$ is a full firing sequence. Since $\sum_{0 \leq i<\left|\sigma^{\prime}\right| c\left(\overrightarrow{\theta_{i}^{\prime}}\right)}<\sum_{0 \leq i<|\sigma| c\left(\vec{\theta}_{i}\right)}$ and the relation between $\sigma$ and $\Theta$, we know that $c\left(\sigma^{\prime}\right)<c(\sigma)$. However, this violates the definition of $\sigma$ being an optimal alignment.

The ILP formulation above allows us to compute an optimal alignment if we know an upper bound $k$ for the length of such an alignment. Unfortunately, such an upper bound cannot be given in advance as this would require knowledge of the alignment sought. Furthermore, the large number of variables in this ILP (the number of transitions in the synchronous product model times the length of the alignment) makes this ILP intractable in any real life setting.

\subsection{Computing Alignments Without Optimality Guarantees}

To overcome the limitations of not knowing the length of the alignment and the intractability of the ILP computation, we introduce an algorithm for incrementally computing alignments. The core idea of this algorithm, which again relies heavily on the marking equation, is the following. We use an ILP problem that constructs an exact prefix of an alignment of relatively short length (for example $x=10$ transitions) and estimates the remainder of the alignment in the same way the $A^{*}$ techniques do. Then, we execute the exact prefix of relatively small length $x$, compute the resulting marking and repeat the computation until we reach the target marking.

Definition 8 ( $k$ of $x$ prefix Alignment as ILP problem). Let $N=\langle P, T, \mathcal{F}\rangle$ be a synchronous product Petri net where $T=T^{s} \cup T^{l} \cup T^{m}$ are the partitions of $T$ and let $\left(N, m_{\perp}, m^{\top}\right)$ a corresponding net system. Furthermore let $c: T \rightarrow \mathbb{R}^{+}$a cost function. We assume $k \leq\left|T^{l}\right|$.

Let $\Theta=\left\{\overrightarrow{\theta_{0}}, \ldots, \overrightarrow{\theta_{x}}\right\}$ be a set of $x+1$ vectors of dimension $|T|$ as the optimal solution to the following ILP problem: 


$$
\begin{aligned}
& \underset{\Sigma}{\operatorname{minimize}} \quad \sum_{0 \leq i \leq x} c\left(\overrightarrow{\theta_{i}}\right) \\
& \text { subject to } \quad \overrightarrow{m_{\perp}}+\sum_{0 \leq j \leq x} \mathbf{N} \cdot \overrightarrow{\theta_{j}}=\overrightarrow{m^{\top}} \\
& \sum_{t \in T^{s} \cup T^{l}} \sum_{0 \leq i<x} \theta_{i}(t) \geq k \\
& \forall 0 \leq i<x \quad \vec{\theta}_{i} \cdot \overrightarrow{1}^{T} \leq 1 \\
& \overrightarrow{m_{\perp}}+\sum_{0 \leq j<i} \mathbf{N} \cdot \overrightarrow{\theta_{j}}-\mathbf{N}^{-} \cdot \overrightarrow{\theta_{i}} \geq 0 \\
& \forall_{0<i<x} \quad \overrightarrow{\theta_{i-1}} \cdot \overrightarrow{1}^{T} \geq \vec{\theta}_{i} \cdot \overrightarrow{1}^{T} \\
& C \cdot \overrightarrow{\theta_{x-1}} \cdot \overrightarrow{1}^{T} \geq \overrightarrow{\theta_{x}} \cdot \overrightarrow{1}^{T}
\end{aligned}
$$

An optimal solution to the problem above constitutes a firing sequence $\sigma$ of length $l=\sum_{0 \leq i<x} \vec{\theta}_{i} \cdot \overrightarrow{1}^{T}$ of the net $N$ identical to Definition 7 . Note that the constant $C$ in Equation 13 is a sufficiently large constant, for example $C=|T|^{2}$. A specific value for $C$ can be identified, but this is beyond the scope of the paper.

The difference between Definition 7 and Definition 8 is relatively small, but significant. The added vector $\overrightarrow{\theta_{x}}$ in the solution does not represent a single transition execution. Instead, it represents the "tail" of the alignment, i.e. the resulting firing sequence $\sigma$ is no longer a full firing sequence as it is not guaranteed to reach the target marking. Instead, it reaches some intermediate marking $m$ and $\overrightarrow{\theta_{x}}$ is a vector underestimating the cost for reaching the final marking from $m$ identical to the underestimate function in $A^{*}$ as defined in Definition 6. Once the optimal solution to the ILP is found, the marking $m$ reached after executing $\sigma$ is taken as a new final marking and the problem is reinstantiated with that marking as initial marking.

The second important difference is the $k$ used solely in Equation 9. This equation ensures that $\sigma$ contains at least $k$ transitions from the set of synchronous moves or log moves, i.e. it guarantees progress as it is a property of a synchronous product that there are no loops in the log move and synchronous move possible.

Using the $k$ of $x$ ILP we present the sequential alignment algorithm as Algorithm 1 and using the algorithm outlined in Algorithm 1 we define an $(k, x)$ sequential alignment.

Definition $9((k, x)$ - Sequential Alignment). Let $N=\langle P, T, \mathcal{F}\rangle$ be a synchronous product Petri net where $T=T^{s} \cup T^{l} \cup T^{m}$ are the partitions of $T$ and let $\left(N, m_{\perp}, m^{\top}\right) a$ corresponding net system. $\sigma=\operatorname{Align}\left(N, m_{\perp}, m^{\top}, \inf ,\left|T^{l}\right|, x, k\right)$ is an $(k, x)$ sequential alignment, where $k \leq\left|T^{l}\right|$ and $k \leq x$.

The sequential alignment algorithm is a recursive algorithm. It starts by solving a $k$ of $x$ ILP problem which for which a solution is assumed to exist. After solving the ILP, the solution is compared to the previous estimate (the cost of $\overrightarrow{\theta_{x}}$ ). If the new optimal 


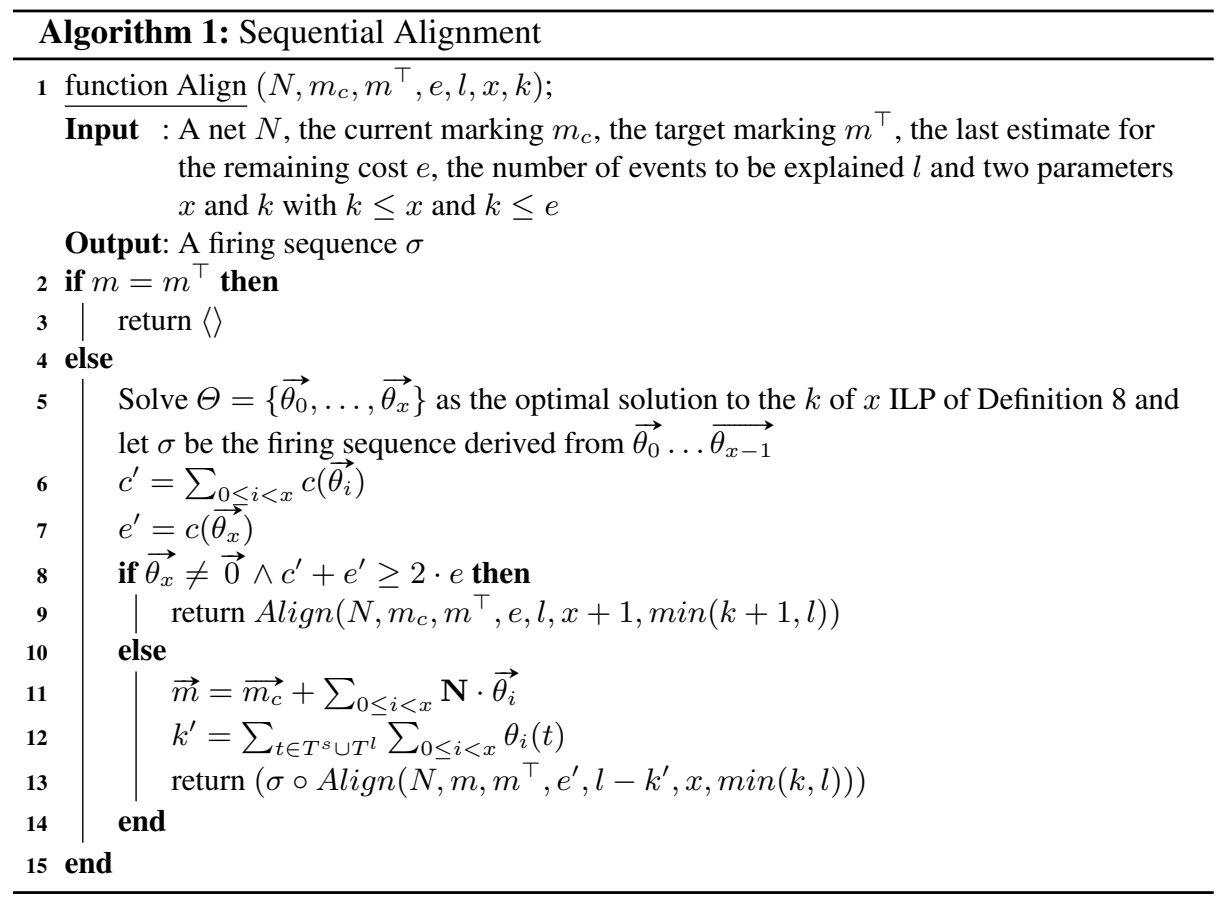

solution deviates too much from the expected solution $e^{\prime}+c^{\prime} \geq 2 \cdot e$ and the $\overrightarrow{\theta_{x}}$ is non zero, i.e. the final marking is not reached, then we go into a backtracking phase. We try again, with increased value of $x$ (and $k$ if applicable). If the initial ILP cannot be solved, i.e. no solution exist, backtracking can also be used. However, we typically assume our process models to be sound workflow models.

It is easy to see that the algorithm terminates, i.e. either the final marking $m^{\top}$ is reached, or the value of $x$ is increased until it equals the length of the shortest path from the current marking to the final marking in which case the solution of the $k$ of $x$ ILP becomes optimal and $\overrightarrow{\theta_{x}}=\overrightarrow{0}$.

\subsection{Quality of Alignments}

The sequential alignment algorithm presented in Algorithm 1 is guaranteed to terminate and to return an alignment. However, it is not guaranteed to return an optimal alignment. This is due to the fact that the marking equation used for the $\overrightarrow{\theta_{x}}$ vector does not correspond to an actual realizable sequence. Instead, as in the original $A^{*}$ approach, is merely underestimates the optimal costs to reach the final marking. As such, sub-optimal decisions may be made in each prefix. In particular, this is the case if the model contains many so-called "transition invariants", the simplest case of which are structured loops of activities.

Even if a trace perfectly fits the model, extreme cases can be devised where the sequential algorithm may construct sub-optimal alignments (although this requires the 
introduction of duplicate labels), while at the same time, for some classes of model and log combinations, optimality can be guaranteed. Hence, overall, it is impossible to say anything about the quality of the delivered alignment in advance. However, as the experiments in the next section show, in practical cases, the alignments are of high quality and the reduced time complexity is well worth the trade-off.

In our experiments, which we present in the next section, we considered the relative error of the costs as a measure for the quality. This relative error is defined as the cost of the sequential alignment exceeding the cost of the optimal alignment as a fraction of the cost of the optimal alignment.

\section{Evaluation}

In order to assess the quality of the proposed technique, we conducted various experiments. In this section, we show one of these experiments on a real-life dataset and model. The dataset used deals with the treatment of sepsis patients in a hospital [9]. There are 1050 cases with in total 15214 events over 16 activities. There are 74 unique sequences of activities in the log and the model used contains 19 labeled transitions and 30 unlabeled routing transitions. The model is free-choice and contains both loops and parallel constructs, i.e. it belongs to the class of models considered in this paper.

The experiments were conducted on a Core i7-4700MQ CPU with 16GB of memory, of which at most $8 \mathrm{~GB}$ of memory were allocated to the Java virtual machine. In the interest of fairness, all algorithms were executed in single-threaded mode ${ }^{8}$.

Figure 5 shows the analysis time of aligning this $\log$ on the given model using three techniques, namely (1) the baseline traditional $A^{*}$, (2) our approach using Gurobi [10] as a backend ILP solver and (3) our approach using LpSolve [11] as a backend solver. The $\mathrm{x}$-axis shows the fitness of the trace (based on the baseline which guarantees optimal alignments) and for each trace, both computation time and relative error in total costs for the alignment returned are plotted. The time is plotted on the left-hand, logarithmic axis and the error on the right-hand axis.

As shown in Figure 5, the computation time of alignments using our approach is orders of magnitude lower than when using $A^{*}$. However, in some cases, suboptimal solutions may be returned which are up to $84 \%$ off in terms of the total costs. The overall error on the entire $\log$ is $7,87 \%$ for Gurobi and $7,05 \%$ for LpSolve. The differences between the two solvers are explained by their local decisions for optimal solutions which may lead to different choices in the alignments. For two other models in the same collection, the results are even better, with at most an $6.7 \%$ cost overestimation.

Figure 5 suggests that, when cases become more fitting, the computation becomes more expensive. However, this result is misleading as the numbers are not corrected for the length of traces, i.e. the traces that are better fitting in this dataset are typically longer. Therefore, in Figure 6 we show the relation between the trace length and the computation time for both $A^{*}$ and for our approach using Gurobi.

\footnotetext{
${ }^{8}$ The classical $A^{*}$ approach can be executed in multi-threaded mode, in which case multiple traces are aligned at once. Furthermore, the Gurobi solver can also be used in multi-threaded mode, which only affects the branch-and-bound phase of the solving.
} 


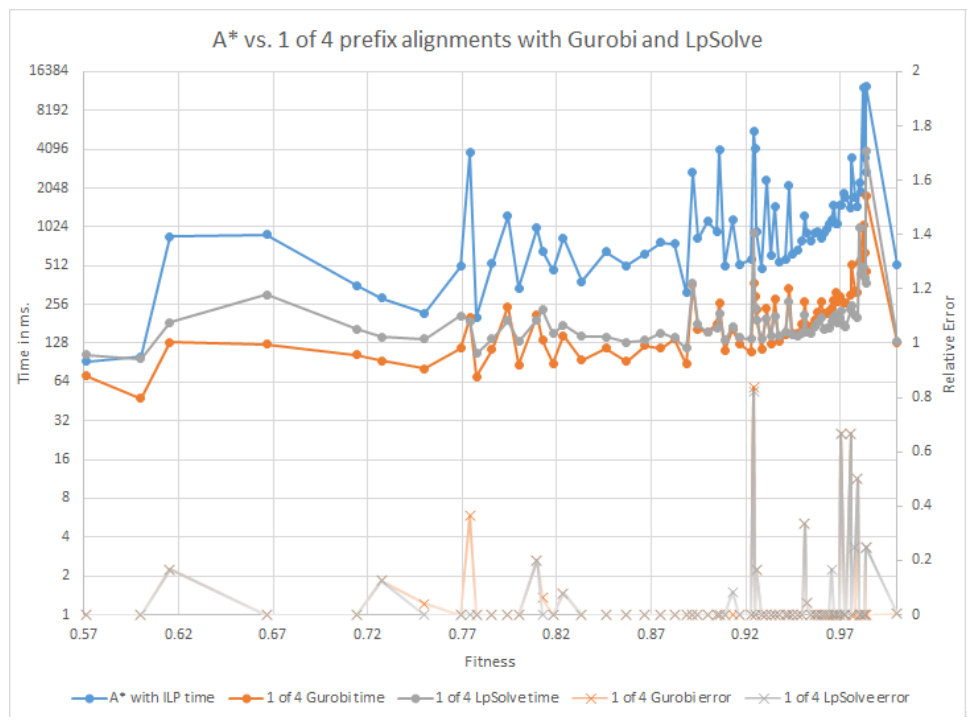

Fig. 5. Comparison of computation time and error of $A^{*}$ with 1-of-4 alignments.

Figure 6 clearly shows that our approach scales linearly in the length of the trace. This is due to the fact that for longer traces, more ILPs need to be solved. However, these ILPs are all of equal size. In the $A^{*}$ case, we see that there is a considerably larger influence of the trace length to the time do compute alignments. This is due to parallelism in the model, as longer traces introduce more and more states that need to be considered by the $A^{*}$ approach.

To emphasize the importance of our work even further, we show results on a wellknown, artificial benchmark example in Figure 7. This example was taken from [12] where a model is presented with 239 uniquely labeled transitions and massive parallelism. Here, we clearly see that our approach, both using LpSolve or Gurobi, can be used to find alignments for all traces within a couple of seconds. The $A^{*}$ approach however, can only find alignments in some cases, before running out of time (the limit per trace was set at 200000 states, roughly corresponding to 15 minutes of computation time). Furthermore, in those cases where the $A^{*}$ completes, our sequential algorithms returns optimal alignments.

In all experiments above, the cost function used was chosen in such a way that the penalties for labeling an event as a so-called log move or a transition as a so-called model move were equal to 1 and all figures were made using 1-of-4 prefix alignments. We tested various other values for both $k$ and $x$ and the results were comparable as long as $k$ is significantly smaller than $x$. The full code is available in the anti-alignment package in ProM and is fully integrated in the conformance checking framework therein. 


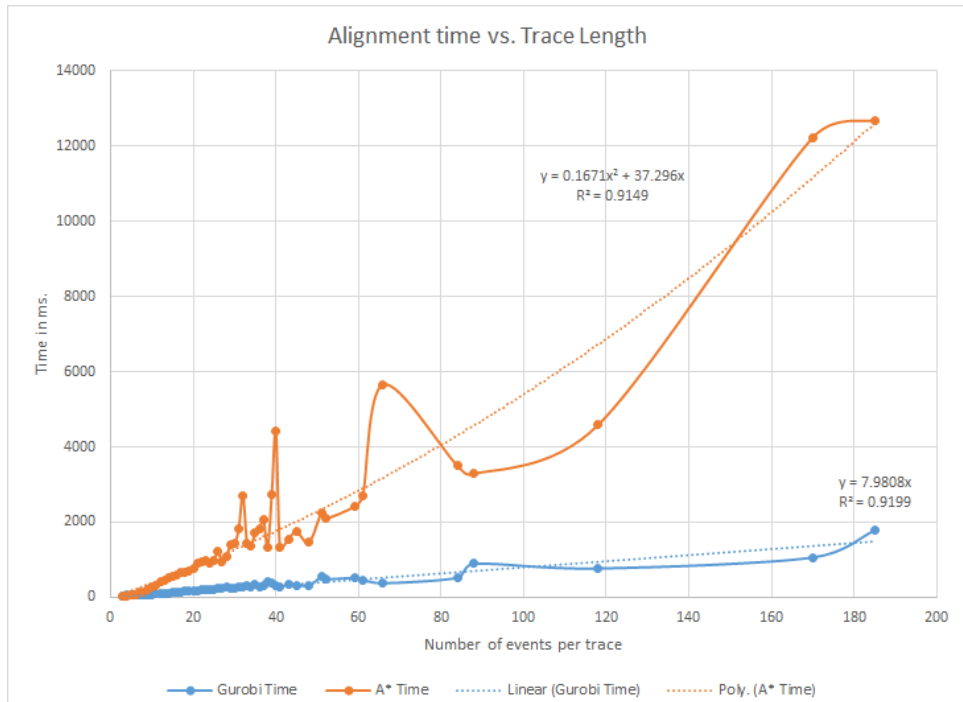

Fig. 6. Time to compute alignments vs. length of the original trace.

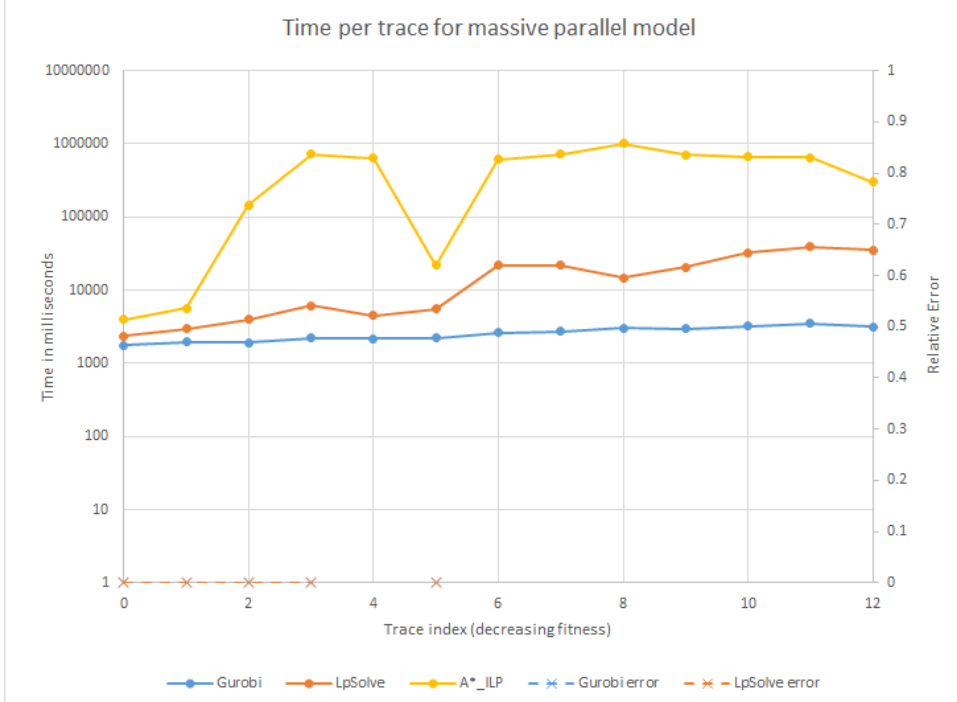

Fig. 7. Comparison of computation time and error of $A^{*}$ with 1-of-4 alignments.

\section{Conclusions}

Alignments are a well-known basis for further analysis when comparing process models to event logs, but traditional alignment techniques suffer from computational complex- 
ity and the unpredictable nature of the computation time. In this paper, we presented an incremental approach to compute alignments for a given log and model using ILP.

Our approach is heuristic in nature, i.e. the result is not guaranteed to be optimal, but the computation time is shown to be linear in the length of the input trace (around $8 \mathrm{~ms}$ per event in our experiments on a high-end laptop computer) and the error in the final results, while depending on the parameters, is shown to be reasonable.

In the paper, we introduce the theoretical foundations of our work, we present the algorithm with proof of termination and we show experimental results on real-life cases. We compare our implementation using both a freely available ILP solver as well as an industrial ILP solver with the state-of-the-art in alignment computation.

All datasets and implementations used in this paper are freely available for download and the software is integrated in the process mining tool ProM.

\section{References}

1. van der Aalst, W.M.P.: Process Mining - Discovery, Conformance and Enhancement of Business Processes. Springer (2011)

2. Adriansyah, A.: Aligning observed and modeled behavior. PhD thesis, Technische Universiteit Eindhoven (2014)

3. Adriansyah, A., Munoz-Gama, J., Carmona, J., van Dongen, B.F., van der Aalst, W.M.P.: Measuring precision of modeled behavior. Inf. Syst. E-Business Management 13(1) (2015) $37-67$

4. Taymouri, F., Carmona, J.: A recursive paradigm for aligning observed behavior of large structured process models. In: Business Process Management - 14th International Conference, BPM 2016, Rio de Janeiro, Brazil, September 18-22, 2016. Proceedings. (2016) 197-214

5. van der Aalst, W.M.P.: Decomposing Petri nets for process mining: A generic approach. Distributed and Parallel Databases 31(4) (2013) 471-507

6. Munoz-Gama, J., Carmona, J., van der Aalst, W.M.P.: Single-entry single-exit decomposed conformance checking. Inf. Syst. 46 (2014) 102-122

7. Murata, T.: Petri nets: Properties, analysis and applications. Proceedings of the IEEE 77(4) (April 1989) 541-574

8. Silva, M., Teruel, E., Colom, J.M.: Linear algebraic and linear programming techniques for the analysis of place/transition net systems. In Reisig, W., Rozenberg, G., eds.: Lecture Notes in Computer Science: Lectures on Petri Nets I: Basic Models. Volume 1491. Springer-Verlag (1998) 309-373

9. Mannhardt, F.: Sepsis dataset (to appear) (2016)

10. Gurobi Optimization, I.: Gurobi optimizer reference manual (2016)

11. Berkelaar, M., Eikland, K., Notebaert, P.: lpsolve : Open source (Mixed-Integer) Linear Programming system

12. Munoz-Gama, J., Carmona, J., van der Aalst, W.M.P.: Conformance checking in the large: Partitioning and topology. In Daniel, F., Wang, J., Weber, B., eds.: Business Process Management - 11th International Conference, BPM 2013, Beijing, China, August 26-30, 2013. Proceedings. Volume 8094 of Lecture Notes in Computer Science., Springer (2013) 130-145 